\title{
Uniformly convex operators and martingale type
}

Jörg Wenzel

\begin{abstract}
The concept of uniform convexity of a Banach space was generalized to linear operators between Banach spaces and studied by Beauzamy [1]. Under this generalization, a Banach space $X$ is uniformly convex if and only if its identity map $I_{X}$ is. Pisier showed that uniformly convex Banach spaces have martingale type $p$ for some $p>1$. We show that this fact is in general not true for linear operators. To remedy the situation, we introduce the new concept of martingale subtype and show, that it is equivalent, also in the operator case, to the existence of an equivalent uniformly convex norm on $X$. In the case of identity maps it is also equivalent to having martingale type $p$ for some $p>1$.

Our main method is to use sequences of ideal norms defined on the class of all linear operators and to study the factorization of the finite summation operators. There is a certain analogy with the theory of Rademacher type.
\end{abstract}

\section{Introduction}

Banach spaces admitting an equivalent uniformly convex norm enjoy several equivalent characterizations. Among others, they are the superreflexive Banach spaces, i. e. not only is such a Banach space $X$ reflexive, but every Banach space whose finite dimensional subspaces can be found (uniformly) in $X$ is reflexive.

A connection with martingales was studied by Pisier [14]. For $1<p \leq 2$, a Banach space $X$ has martingale type $p$ if there exists a constant $c \geq 0$

2000 Mathematics Subject Classification: Primary 46B03; Secondary 47A30, 46B07.

Keywords: Banach spaces, linear operators, martingale type, martingale subtype, superreflexivity, uniform convexity, summation operator. 
such that

$$
\left(\int_{0}^{1}\left\|\sum_{k=1}^{n} d_{k}(t)\right\|^{p} d t\right)^{1 / p} \leq c\left(\sum_{k=1}^{n} \int_{0}^{1}\left\|d_{k}(t)\right\|^{p} d t\right)^{1 / p}
$$

for all $X$-valued martingale difference sequences $d_{1}, \ldots, d_{n}$.

For our purpose the fundamental result of Pisier's paper [14, Thm. 3.2, p. 340] combined with James's and Enflo's investigations [10, Thm. 4, p. 903], [6, p. 281] can be summarized as follows. (See further down for detailed definitions.)

Theorem 1. For a Banach space $X$ the following properties are equivalent:

(i) $X$ has martingale type $p$ for some $p>1$,

(ii) $X$ is superreflexive,

(iii) $X$ admits an equivalent uniformly convex norm,

(iv) $X$ does not factor the finite summation operators $\Sigma_{n}$ uniformly.

If one is interested in linear operators between Banach spaces instead of just Banach spaces, the above theorem is no longer true, as can be seen by examples (see Example 3 at the end). The aim of this paper is to prove an operator-theoretic replacement of Theorem 1; see Theorem 2 in Section 5.

The main difficulties arising in this setting are the lack of a suitable substitute of J-convexity in the operator case (see Beauzamy [2, p. 265] for a definition of J-convexity) and the fact that the submultiplicativity of the martingale type ideal norms can no longer be exploited.

The following general method has turned out to be useful to generalize Banach space-theoretic results to results about operators.

Given a sequence of parameters $\left(\boldsymbol{\alpha}_{n}\right)$ associating with every operator $T: X \rightarrow Y$ a sequence of non-negative numbers $\left(\boldsymbol{\alpha}_{n}(T)\right)$, let $\boldsymbol{\alpha}_{n}(\infty):=$ $\sup \boldsymbol{\alpha}_{n}(T)$, where the supremum is taken over all operators $T: X \rightarrow Y$ of norm 1 and all Banach spaces $X$ and $Y$. Then the sequence $\left(\boldsymbol{\alpha}_{n}(\infty)\right)$ describes the 'worst' behavior that can occur for an operator $T$. In the Banach space case, one is mostly interested in the boundedness of the sequence $\left(\boldsymbol{\alpha}_{n}\left(I_{X}\right)\right)$ of the identity map of a Banach space $X$, i. e. $\boldsymbol{\alpha}_{n}\left(I_{X}\right)=O(1)$. In the operator case, the behavior $\boldsymbol{\alpha}_{n}(T) / \boldsymbol{\alpha}_{n}(\infty) \rightarrow 0$, i. e. $\boldsymbol{\alpha}_{n}(T)=o\left(\boldsymbol{\alpha}_{n}(\infty)\right)$ is much more useful. Another beautiful example for this heuristic in the context of Rademacher and Gauss type is given by Hinrichs in [9].

In particular, taking as $\boldsymbol{\alpha}_{n}(T)$ the martingale type ideal norm $\boldsymbol{\tau}_{n}(T)$ formed with $n$ martingale differences, the condition $\boldsymbol{\tau}_{n}(T)=o(\sqrt{n})$ will be the right replacement for Condition (i) in Theorem 1. 
Let us quickly review the contents of this article. In Section 2 we introduce martingale and Haar (co)type ideal norms, which are close relatives. The main result of this section is that all the $o$-conditions described above for these four types of ideal norms yield equivalent properties. In Section 3 we establish the connection of the martingale type ideal norms with the factorization of the finite summation operators. To do so, we use a variant of the martingale type ideal norms, namely the equal norm martingale type ideal norms. In Section 4 we repeat the definitions of uniform convexity and uniform smoothness of linear operators, introduce the super weakly compact operators and give various characterizations due to Beauzamy [1] connecting the two concepts. Since the main emphasis of this article is on the connection with martingales, we are rather brief here and give mostly references for the proofs. Finally in Section 5 we formulate and prove our main theorem and provide an example of an operator, for which Theorem 1 is false.

To finish this introduction, let us point out some notational conventions used throughout. We write $B_{X}$ for the unit ball of a Banach space $X$ and $I_{X}$ for its identity map. Furthermore, the reader has already realized, that we use Landau's big- $O$ and little- $O$ notation.

\section{Martingale type and cotype}

First of all, we introduce the martingale type and cotype ideal norms. They were first considered by Pisier in [14, Rem. 3.3, p. 346]. We also consider Haar type and cotype ideal norms as restrictions of martingale type and cotype ideal norms to special classes of martingales. An operator is said to have the corresponding subtype or subcotype, if these sequences of ideal norms behave just a little better than the worst case. The main result of this section will be that all four possible subtype and subcotype properties coincide.

Let us start by considering an arbitrary martingale $\left(f_{k}\right)$ defined on $[0,1)$ with values in a Banach space $X$ and adapted to a filtration $\mathcal{F}_{0} \subseteq \mathcal{F}_{1} \subseteq \ldots$ of finitely generated $\sigma$-algebras. We denote by $E_{k}$ the operator of conditional expectation with respect to the $\sigma$-algebra $\mathcal{F}_{k}$.

In particular, taking for $\mathcal{F}_{k}$ the $\sigma$-algebra generated by the dyadic intervals

$$
\Delta_{k}^{(j)}:=\left[\frac{j-1}{2^{k}}, \frac{j}{2^{k}}\right),
$$

we obtain the so called Walsh-Paley or dyadic martingales. Since the corresponding martingales $f_{n}$ are just linear combinations of the Haar functions 
$\chi_{k}^{(j)}$ for $k=0, \ldots, n$ and $j=1, \ldots, 2^{k-1}$, we also use the term Haar polynomials to denote the functions $f_{n}$ in this case. Remember, that the Haar functions $\chi_{k}^{(j)}$ are defined by

$$
\chi_{k}^{(j)}(t):= \begin{cases}+2^{(k-1) / 2} & \text { if } t \in \Delta_{k}^{(2 j-1)}, \\ -2^{(k-1) / 2} & \text { if } t \in \Delta_{k}^{(2 j)}, \\ 0 & \text { otherwise. }\end{cases}
$$

We let $\chi_{0}^{(0)} \equiv 1$.

We will mainly deal with the sequence of martingale differences $\left(d_{k}\right)$ instead of $\left(f_{k}\right)$, where $d_{1}=f_{1}$ and $d_{k}=f_{k}-f_{k-1}$ for $k>1$. The Banach space of square integrable $X$-valued functions on $[0,1)$ is denoted by $\left[L_{2}, X\right]$. For $f \in\left[L_{2}, X\right]$ we write

$$
\left\|f \mid L_{2}\right\|:=\left(\int_{0}^{1}\|f(t)\|^{2} d t\right)^{1 / 2} .
$$

The fact that $\left\|E_{k} f\left|L_{2}\|\leq\| f\right| L_{2}\right\|$ for all $f \in\left[L_{2}, X\right]$ and $k \in \mathbb{N}$ will be frequently used.

With each operator $T: X \rightarrow Y$ we associate the operator $\left[L_{2}, T\right]$ : $\left[L_{2}, X\right] \rightarrow\left[L_{2}, Y\right]$, defined by

$$
\left[L_{2}, T\right] f(t):=T(f(t)) .
$$

For any two functions $f \in\left[L_{2}, X\right]$ and $g^{\prime} \in\left[L_{2}, Y^{\prime}\right]$ we write

$$
\left\langle\left[L_{2}, T\right] f, g^{\prime}\right\rangle:=\int_{0}^{1}\left\langle T f(t), g^{\prime}(t)\right\rangle d t .
$$

Definition. For $T: X \rightarrow Y$, the $n$-th martingale type ideal norm $\boldsymbol{\tau}\left(T \mid \mathcal{M}_{n}\right)$ is the smallest constant $c \geq 0$ such that

$$
\left\|\sum_{k=1}^{n}\left[L_{2}, T\right] d_{k} \mid L_{2}\right\| \leq c\left(\sum_{k=1}^{n}\left\|d_{k} \mid L_{2}\right\|^{2}\right)^{1 / 2}
$$

for all $X$-valued martingale difference sequences $d_{1}, \ldots, d_{n}$ adapted to any filtration on $[0,1)$.

The $n$-th martingale cotype ideal norm $\gamma\left(T \mid \mathcal{M}_{n}\right)$ is the smallest constant $c \geq 0$ such that

$$
\left(\sum_{k=1}^{n}\left\|\left[L_{2}, T\right] d_{k} \mid L_{2}\right\|^{2}\right)^{1 / 2} \leq c\left\|\sum_{k=1}^{n} d_{k} \mid L_{2}\right\|
$$

for all $X$-valued martingale difference sequences $d_{1}, \ldots, d_{n}$ adapted to any filtration on $[0,1)$. 
Note that, for norm one operators, both sequences, $\left(\boldsymbol{\tau}\left(T \mid \mathcal{M}_{n}\right)\right)$ and $\left(\gamma\left(T \mid \mathcal{M}_{n}\right)\right)$, are bounded by $2 \sqrt{n}$ and therefore the following definition makes sense.

Definition. An operator $T: X \rightarrow Y$ has martingale subtype or martingale subcotype if $\boldsymbol{\tau}\left(T \mid \mathcal{M}_{n}\right) / \sqrt{n} \rightarrow 0$ or $\gamma\left(T \mid \mathcal{M}_{n}\right) / \sqrt{n} \rightarrow 0$, respectively.

For convenience we introduce the following notation for the dyadic trees:

$$
\mathbb{D}_{m}^{n}:=\left\{(k, j): k=m, \ldots, n ; j=1, \ldots, 2^{k-1}\right\} \quad \text { for } 1 \leq m \leq n .
$$

We let $\mathbb{D}_{0}^{n}:=\mathbb{D}_{1}^{n} \cup\{(0,0)\}$.

Definition. For $T: X \rightarrow Y$, the Haar type ideal norm $\boldsymbol{\tau}\left(T \mid \mathcal{H}\left(\mathbb{D}_{m}^{n}\right)\right)$ associated with the index set $\mathbb{D}_{m}^{n}$ is the smallest constant $c \geq 0$ such that

$$
\left\|\sum_{\mathbb{D}_{m}^{n}} T x_{k}^{(j)} \chi_{k}^{(j)} \mid L_{2}\right\| \leq c\left(\sum_{\mathbb{D}_{m}^{n}}\left\|x_{k}^{(j)}\right\|^{2}\right)^{1 / 2}
$$

for all $\left(x_{k}^{(j)}\right) \subseteq X$.

The Haar cotype ideal norm $\gamma\left(T \mid \mathcal{H}\left(\mathbb{D}_{m}^{n}\right)\right)$ associated with the index set $\mathbb{D}_{m}^{n}$ is the smallest constant $c \geq 0$ such that

$$
\left(\sum_{\mathbb{D}_{m}^{n}}\left\|T x_{k}^{(j)}\right\|^{2}\right)^{1 / 2} \leq c\left\|\sum_{\mathbb{D}_{m}^{n}} x_{k}^{(j)} \chi_{k}^{(j)} \mid L_{2}\right\|
$$

for all $\left(x_{k}^{(j)}\right) \subseteq X$

As before, we will be interested in the suboptimal behavior of these sequences.

Definition. An operator $T: X \rightarrow Y$ has Haar subtype or Haar subcotype if $\boldsymbol{\tau}\left(T \mid \mathcal{H}\left(\mathbb{D}_{0}^{n}\right)\right) / \sqrt{n} \rightarrow 0$ or $\gamma\left(T \mid \mathcal{H}\left(\mathbb{D}_{0}^{n}\right)\right) / \sqrt{n} \rightarrow 0$, respectively.

It is easily verified that

$$
\begin{aligned}
& \boldsymbol{\tau}\left(T \mid \mathcal{H}\left(\mathbb{D}_{1}^{n}\right)\right) \leq \boldsymbol{\tau}\left(T \mid \mathcal{H}\left(\mathbb{D}_{0}^{n}\right)\right) \leq 2 \boldsymbol{\tau}\left(T \mid \mathcal{H}\left(\mathbb{D}_{1}^{n}\right)\right) \\
& \boldsymbol{\gamma}\left(T \mid \mathcal{H}\left(\mathbb{D}_{1}^{n}\right)\right) \leq \boldsymbol{\gamma}\left(T \mid \mathcal{H}\left(\mathbb{D}_{0}^{n}\right)\right) \leq 3 \boldsymbol{\gamma}\left(T \mid \mathcal{H}\left(\mathbb{D}_{1}^{n}\right)\right)
\end{aligned}
$$

Since for any $\left(x_{k}^{(j)}\right) \subseteq X$ the sequence of functions $d_{k}:=\sum_{j=1}^{2^{k-1}} x_{k}^{(j)} \chi_{k}^{(j)}$ forms a sequence of martingale differences, we obviously have

$$
\boldsymbol{\tau}\left(T \mid \mathcal{H}\left(\mathbb{D}_{1}^{n}\right)\right) \leq \boldsymbol{\tau}\left(T \mid \mathcal{M}_{n}\right) \quad \text { and } \quad \gamma\left(T \mid \mathcal{H}\left(\mathbb{D}_{1}^{n}\right)\right) \leq \gamma\left(T \mid \mathcal{M}_{n}\right)
$$

No reverse estimate is known. In the limiting case where one of these sequences of ideal norms behaves like $o(\sqrt{n})$ we have, however, equivalence. 
Proposition 1. For any operator $T: X \rightarrow Y$ the following properties are equivalent:

(i) T has martingale subtype,

(ii) $T$ has martingale subcotype,

(iii) T has Haar subtype,

(iv) T has Haar subcotype.

We postpone the proof of Proposition 1 in order to provide some prerequisites. The main idea of the proof is contained in the following lemma.

Lemma 1. Let $T: X \rightarrow Y$, then we have

$$
\frac{\boldsymbol{\tau}\left(T \mid \mathcal{M}_{2^{n}}\right)}{2^{n / 2}} \leq 3 \frac{\gamma\left(T \mid \mathcal{H}\left(\mathbb{D}_{0}^{n}\right)\right)}{n^{1 / 2}}
$$

Proof. Given any sequence $\left(d_{1}, \ldots, d_{2^{n}}\right)$ of $X$-valued martingale differences, define a function $F_{n}:[0,1) \times[0,1) \rightarrow X$ by $F_{n}(s, t):=d_{i}(t)$ if $s \in \Delta_{n}^{(i)}$.

Obviously

$$
\int_{0}^{1} \int_{0}^{1}\left\|F_{n}(s, t)\right\|^{2} d t d s=\sum_{i=1}^{2^{n}} \int_{\Delta_{n}^{(i)}} \int_{0}^{1}\left\|F_{n}(s, t)\right\|^{2} d t d s=\frac{1}{2^{n}} \sum_{i=1}^{2^{n}}\left\|d_{i} \mid L_{2}\right\|^{2} .
$$

Viewing $F_{n}(\cdot, t)$ as a Haar polynomial, we have

$$
F_{n}(s, t)=\sum_{\mathbb{D}_{0}^{n}} x_{k}^{(j)}(t) \chi_{k}^{(j)}(s)
$$

where the functions $x_{k}^{(j)}$ are defined by $x_{k}^{(j)}(t):=\int_{0}^{1} F_{n}(s, t) \chi_{k}^{(j)}(s) d s$. By the definition of $\gamma\left(T \mid \mathcal{H}\left(\mathbb{D}_{0}^{n}\right)\right)$ we have

$$
\sum_{\mathbb{D}_{0}^{n}}\left\|T x_{k}^{(j)}(t)\right\|^{2} \leq \gamma\left(T \mid \mathcal{H}\left(\mathbb{D}_{0}^{n}\right)\right)^{2}\left\|\sum_{\mathbb{D}_{0}^{n}} x_{k}^{(j)}(t) \chi_{k}^{(j)} \mid L_{2}\right\|^{2}
$$

Integration with respect to $t \in[0,1)$ yields

$$
\begin{aligned}
\sum_{\mathbb{D}_{0}^{n}}\left\|\left[L_{2}, T\right] x_{k}^{(j)} \mid L_{2}\right\|^{2} & \leq \gamma\left(T \mid \mathcal{H}\left(\mathbb{D}_{0}^{n}\right)\right)^{2}\left\|F_{n} \mid L_{2}\right\|^{2} \\
& =\gamma\left(T \mid \mathcal{H}\left(\mathbb{D}_{0}^{n}\right)\right)^{2} \frac{1}{2^{n}} \sum_{i=1}^{2^{n}}\left\|d_{i} \mid L_{2}\right\|^{2}
\end{aligned}
$$


Letting $\mathbb{N}_{k}^{(j)}:=\left\{i: \Delta_{n}^{(i)} \subseteq \Delta_{k}^{(j)}\right\}$ and observing that $\mathbb{N}_{k-1}^{(j)}=\mathbb{N}_{k}^{(2 j-1)} \cup$ $\mathbb{N}_{k}^{(2 j)}$, we obtain for $x_{k}^{(j)}$

$$
\begin{aligned}
x_{k}^{(j)}(t) & =\int_{0}^{1} F_{n}(s, t) \chi_{k}^{(j)}(s) d s=2^{(k-1) / 2}\left(\int_{\Delta_{k}^{(2 j-1)}} F_{n}(s, t) d s-\int_{\Delta_{k}^{(2 j)}} F_{n}(s, t) d s\right) \\
& =2^{(k-1) / 2-n}\left(\sum_{\mathbb{N}_{k}^{(2 j-1)}} d_{i}(t)-\sum_{\mathbb{N}_{k}^{(2 j)}} d_{i}(t)\right) .
\end{aligned}
$$

This implies that

$$
\left\|\left[L_{2}, T\right] x_{k}^{(j)}\left|L_{2}\left\|=2^{(k-1) / 2-n}\right\| \sum_{\mathbb{N}_{k}^{(2 j-1)}}\left[L_{2}, T\right] d_{i}-\sum_{\mathbb{N}_{k}^{(2 j)}}\left[L_{2}, T\right] d_{i}\right| L_{2}\right\| .
$$

Since the conditional expectation operator has norm one in $\left[L_{2}, X\right]$ it now follows that

$$
\left\|\sum_{\mathbb{N}_{k}^{(2 j-1)}}\left[L_{2}, T\right] d_{i}\left|L_{2}\|\leq\| \sum_{\mathbb{N}_{k}^{(2 j-1)}}\left[L_{2}, T\right] d_{i}-\sum_{\mathbb{N}_{k}^{(2 j)}}\left[L_{2}, T\right] d_{i}\right| L_{2}\right\|
$$

and therefore by the triangle inequality

$$
\begin{aligned}
\left\|\sum_{\mathbb{N}_{k-1}^{(j)}}\left[L_{2}, T\right] d_{i} \mid L_{2}\right\| & \leq\left\|\sum_{\mathbb{N}_{k}^{(2 j-1)}}\left[L_{2}, T\right] d_{i}-\sum_{\mathbb{N}_{k}^{(2 j)}}\left[L_{2}, T\right] d_{i}\left|L_{2}\|+2\| \sum_{\mathbb{N}_{k}^{(2 j-1)}}\left[L_{2}, T\right] d_{i}\right| L_{2}\right\| \\
& \leq 3\left\|\sum_{\mathbb{N}_{k}^{(2 j-1)}}\left[L_{2}, T\right] d_{i}-\sum_{\mathbb{N}_{k}^{(2 j)}}\left[L_{2}, T\right] d_{i} \mid L_{2}\right\| .
\end{aligned}
$$

Using (2.2), (2.3) and (2.4) the proof completes as follows:

$$
\begin{aligned}
& \left\|\sum_{i=1}^{2^{n}}\left[L_{2}, T\right] d_{i} \mid L_{2}\right\|=\left(\frac{1}{n} \sum_{k=1}^{n}\left\|\sum_{j=1}^{2^{k-1}} \sum_{\mathbb{N}_{k-1}^{(j)}}\left[L_{2}, T\right] d_{i} \mid L_{2}\right\|^{2}\right)^{1 / 2} \\
& \leq\left(\frac{1}{n} \sum_{k=1}^{n}\left(\sum_{j=1}^{2^{k-1}}\left\|\sum_{\mathbb{N}_{k-1}^{(j)}}\left[L_{2}, T\right] d_{i} \mid L_{2}\right\|\right)^{2}\right)^{1 / 2} \\
& \leq 3\left(\frac{1}{n} \sum_{k=1}^{n} 2^{k-1} \sum_{j=1}^{2^{k-1}}\left\|\sum_{\mathbb{N}_{k}^{(2 j-1)}}\left[L_{2}, T\right] d_{i}-\sum_{\mathbb{N}_{k}^{(2 j)}}\left[L_{2}, T\right] d_{i} \mid L_{2}\right\|^{2}\right)^{1 / 2} \\
& \leq 3\left(\frac{2^{2 n}}{n} \sum_{k=1}^{n} \sum_{j=1}^{2^{k-1}}\left\|\left[L_{2}, T\right] x_{k}^{(j)} \mid L_{2}\right\|^{2}\right)^{1 / 2} \leq 3 \gamma\left(T \mid \mathcal{H}\left(\mathbb{D}_{0}^{n}\right)\right)\left(\frac{2^{n}}{n} \sum_{i=1}^{2^{n}}\left\|d_{i} \mid L_{2}\right\|^{2}\right)^{1 / 2}
\end{aligned}
$$


We next observe that the martingale and Haar type and cotype ideal norms are dual to each other.

Proposition 2. For $0 \leq m<n$ we have

$$
\begin{aligned}
\gamma\left(T \mid \mathcal{H}\left(\mathbb{D}_{m}^{n}\right)\right) & \leq \boldsymbol{\tau}\left(T^{\prime} \mid \mathcal{H}\left(\mathbb{D}_{m}^{n}\right)\right) \leq 2 \gamma\left(T \mid \mathcal{H}\left(\mathbb{D}_{m}^{n}\right)\right) \\
\gamma\left(T^{\prime} \mid \mathcal{H}\left(\mathbb{D}_{m}^{n}\right)\right) & \leq \boldsymbol{\tau}\left(T \mid \mathcal{H}\left(\mathbb{D}_{m}^{n}\right)\right) \leq 2 \gamma\left(T^{\prime} \mid \mathcal{H}\left(\mathbb{D}_{m}^{n}\right)\right)
\end{aligned}
$$

If $m=0$, we can omit the factors 2 and have equality. On the other hand

$$
\begin{aligned}
& \gamma\left(T \mid \mathcal{M}_{n}\right) \leq 2 \boldsymbol{\tau}\left(T^{\prime} \mid \mathcal{M}_{n}\right) \leq 4 \gamma\left(T \mid \mathcal{M}_{n}\right) \\
& \gamma\left(T^{\prime} \mid \mathcal{M}_{n}\right) \leq 2 \boldsymbol{\tau}\left(T \mid \mathcal{M}_{n}\right) \leq 4 \gamma\left(T^{\prime} \mid \mathcal{M}_{n}\right)
\end{aligned}
$$

Proof. The proof can be obtained using standard duality techniques and is left to the reader.

We can now prove Proposition 1.

Proof. It follows from Lemma 1 and (2.1) that

$$
\frac{\boldsymbol{\tau}\left(T \mid \mathcal{M}_{2^{n}}\right)}{2^{n / 2}} \leq 3 \frac{\gamma\left(T \mid \mathcal{H}\left(\mathbb{D}_{0}^{n}\right)\right)}{n^{1 / 2}} \leq 6 \frac{\left.\gamma\left(T \mid \mathcal{M}_{n}\right)\right)}{n^{1 / 2}}
$$

Since the same is true for $T^{\prime}$, it follows from Proposition 2 that

$$
\frac{\gamma\left(T \mid \mathcal{M}_{2^{n}}\right)}{2^{n / 2}} \leq 6 \frac{\boldsymbol{\tau}\left(T \mid \mathcal{H}\left(\mathbb{D}_{0}^{n}\right)\right)}{n^{1 / 2}} \leq 12 \frac{\left.\boldsymbol{\tau}\left(T \mid \mathcal{M}_{n}\right)\right)}{n^{1 / 2}}
$$

Hence if one of these quotients tends to zero, all the others tend to zero too, which proves the proposition by virtue of the monotonicity of the involved ideal norms.

\section{Equal norm martingale type}

Definition. For $T: X \rightarrow Y$, the $n$-th equal norm martingale type ideal norm $\boldsymbol{\tau}^{\circ}\left(T \mid \mathcal{M}_{n}\right)$ is the smallest constant $c \geq 0$ such that

$$
\left\|\sum_{k=1}^{n}\left[L_{2}, T\right] d_{k} \mid L_{2}\right\| \leq c\left(\sum_{k=1}^{n}\left\|d_{k} \mid L_{2}\right\|^{2}\right)^{1 / 2}
$$

for all $X$-valued martingale difference sequences $d_{1}, \ldots, d_{n}$ adapted to any filtration on $[0,1)$ under the additional assumption that $\left\|d_{1} \mid L_{2}\right\|=\cdots=$ $\left\|d_{n} \mid L_{2}\right\|$.

The quantities $\boldsymbol{\tau}^{\circ}\left(T \mid \mathcal{M}_{n}\right)$ can also be defined in a different way. 
Lemma 2. For $T: X \rightarrow Y$, the ideal norm $\boldsymbol{\tau}^{\circ}\left(T \mid \mathcal{M}_{n}\right)$ is the smallest constant $c \geq 0$ such that

$$
\left\|\sum_{k=1}^{n}\left[L_{2}, T\right] d_{k}\left|L_{2}\left\|\leq c n^{1 / 2} \sup _{k=1, \ldots, n}\right\| d_{k}\right| L_{2}\right\|
$$

for all $X$-valued martingale difference sequences $d_{1}, \ldots, d_{n}$ adapted to any filtration on $[0,1)$.

Proof. For the time being denote by $\boldsymbol{\tau}^{\circ \circ}\left(T \mid \mathcal{M}_{n}\right)$ the smallest constant such that (3.1) holds. It is obvious that for $\left\|d_{1}\left|L_{2}\|=\cdots=\| d_{n}\right| L_{2}\right\|$

$$
\begin{aligned}
\left\|\sum_{k=1}^{n}\left[L_{2}, T\right] d_{k} \mid L_{2}\right\| & \leq \boldsymbol{\tau}^{\circ \circ}\left(T \mid \mathcal{M}_{n}\right) n^{1 / 2} \sup _{k=1, \ldots, n}\left\|d_{k} \mid L_{2}\right\|^{2} \\
& =\boldsymbol{\tau}^{\circ \circ}\left(T \mid \mathcal{M}_{n}\right)\left(\sum_{k=1}^{n}\left\|d_{k} \mid L_{2}\right\|^{2}\right)^{1 / 2} .
\end{aligned}
$$

Therefore $\boldsymbol{\tau}^{\circ}\left(T \mid \mathcal{M}_{n}\right) \leq \boldsymbol{\tau}^{\circ \circ}\left(T \mid \mathcal{M}_{n}\right)$.

On the other hand, let $d_{1}, \ldots, d_{n}$ be an arbitrary sequence of martingale differences. It follows that for $\tilde{d}_{k}:=d_{k} /\left\|d_{k} \mid L_{2}\right\|$

$$
\left\|\sum_{k=1}^{n}\left[L_{2}, T\right] \tilde{d}_{k} \mid L_{2}\right\| \leq \boldsymbol{\tau}^{\circ}\left(T \mid \mathcal{M}_{n}\right) n^{1 / 2} .
$$

But the same is true for $\zeta_{k} \tilde{d}_{k}$ instead of $\tilde{d}_{k}$, where $\left|\zeta_{k}\right|=1$. An extreme point argument then yields that

$$
\left\|\sum_{k=1}^{n}\left[L_{2}, T\right] \alpha_{k} \tilde{d}_{k} \mid L_{2}\right\| \leq \boldsymbol{\tau}^{\circ}\left(T \mid \mathcal{M}_{n}\right) n^{1 / 2}
$$

whenever $\left|\alpha_{k}\right| \leq 1$. In particular, we may take

$$
\alpha_{k}:=\frac{\left\|d_{k} \mid L_{2}\right\|}{\sup _{h=1, \ldots, n}\left\|d_{h} \mid L_{2}\right\|},
$$

which shows that $\boldsymbol{\tau}^{\circ \circ}\left(T \mid \mathcal{M}_{n}\right) \leq \boldsymbol{\tau}^{\circ}\left(T \mid \mathcal{M}_{n}\right)$.

Obviously we have

$$
\boldsymbol{\tau}^{\circ}\left(T \mid \mathcal{M}_{n}\right) \leq \boldsymbol{\tau}\left(T \mid \mathcal{M}_{n}\right) .
$$

The main purpose of this section is to prove a reverse estimate. We follow an approach similar to Bourgain/Kalton/Tzafriri in [3, Thm. 3.1., p. 160] where 
they show that equal norm Rademacher type 2 is equivalent to ordinary Rademacher type 2. The main idea is contained in the following construction of 'glueing' together $m$ copies of a given martingale of length $n$, which yields a martingale of length $m n$ with smaller differences. An appropriate blocking of this longer martingale will then give a martingale of length of order $n$ and nearly equal $L_{2}$-norms.

Let $\phi_{j}^{m}:\left[\frac{j-1}{m}, \frac{j}{m}\right) \rightarrow[0,1)$ be defined by $\phi_{j}^{m}(t):=m t-j+1$. Given a function $f:[0,1) \rightarrow X$, we denote by $\Phi_{j}^{m} f$ the function

$$
\Phi_{j}^{m} f(t):= \begin{cases}f\left(\phi_{j}^{m}(t)\right) & \text { if } t \in\left[\frac{j-1}{m}, \frac{j}{m}\right) \\ 0 & \text { otherwise. }\end{cases}
$$

Given a sequence of martingale differences $\left(d_{k}\right)$, the sequence of martingale differences

$$
\Phi_{1}^{m} d_{1}, \ldots, \Phi_{m}^{m} d_{1}, \Phi_{1}^{m} d_{2}, \ldots, \Phi_{m}^{m} d_{2}, \ldots, \Phi_{1}^{m} d_{n}, \ldots, \Phi_{m}^{m} d_{n}
$$

is adapted to the filtration

$$
\Phi_{1}^{m} \mathcal{F}_{1}, \ldots, \Phi_{m}^{m} \mathcal{F}_{1}, \Phi_{1}^{m} \mathcal{F}_{2}, \ldots, \Phi_{m}^{m} \mathcal{F}_{2}, \ldots, \Phi_{1}^{m} \mathcal{F}_{n}, \ldots, \Phi_{m}^{m} \mathcal{F}_{n}
$$

where $\Phi_{j}^{m} \mathcal{F}_{k}$ is the $\sigma$-algebra generated by all sets $A \subseteq[0,1)$ such that $\phi_{j}^{m}(A) \in \mathcal{F}_{k}$ and by all its predecessor $\sigma$-algebras.

Observe that

$$
\left\|\sum_{k=1}^{n} \sum_{j=1}^{m} \Phi_{j}^{m} d_{k}\left|L_{2}\|=\| \sum_{k=1}^{n} d_{k}\right| L_{2}\right\| \quad \text { and } \quad\left\|\Phi_{j}^{m} d_{k}\left|L_{2}\left\|^{2}=\frac{1}{m}\right\| d_{k}\right| L_{2}\right\|^{2} .
$$

Moreover, all differences $\Phi_{j}^{m} d_{k}$ in any block of length at most $m$ have disjoint support.

Lemma 3. The sequence $\left(\boldsymbol{\tau}^{\circ}\left(T \mid \mathcal{M}_{n}\right)\right)$ is non-decreasing.

Proof. Let $d_{1}, \ldots, d_{n}$ be $X$-valued martingale differences such that $\left\|d_{k} \mid L_{2}\right\|=$ 1 . For $m:=n+1$ the construction above yields a martingale difference sequence of length $n(n+1)$. Define a new sequence of martingale differences $\tilde{d}_{1}, \ldots, \tilde{d}_{n+1}$ by blocking $n$ consecutive terms:

$$
\tilde{d}_{h}:=\Phi_{n-h+3}^{m} d_{h-1}+\cdots+\Phi_{n+1}^{m} d_{h-1}+\Phi_{1}^{m} d_{h}+\ldots \Phi_{n-h+1}^{m} d_{h} .
$$

Since $\left\|\tilde{d}_{h} \mid L_{2}\right\|=\sqrt{\frac{n}{n+1}}$ it follows that

$$
\left\|\sum_{k=1}^{n}\left[L_{2}, T\right] d_{k}\left|L_{2}\|=\| \sum_{h=1}^{n+1}\left[L_{2}, T\right] \tilde{d}_{h}\right| L_{2}\right\| \leq \boldsymbol{\tau}^{\circ}\left(T \mid \mathcal{M}_{n+1}\right)\left(\sum_{k=1}^{n}\left\|d_{k} \mid L_{2}\right\|^{2}\right)^{1 / 2}
$$

which proves that $\boldsymbol{\tau}^{\circ}\left(T \mid \mathcal{M}_{n}\right) \leq \boldsymbol{\tau}^{\circ}\left(T \mid \mathcal{M}_{n+1}\right)$. 
Lemma 4. $\boldsymbol{\tau}\left(T \mid \mathcal{M}_{n}\right) \leq 16 \boldsymbol{\tau}^{\circ}\left(T \mid \mathcal{M}_{n}\right)$.

Proof. Let $d_{1}, \ldots, d_{n}$ be $X$-valued martingale differences. By scaling we may assume that $\sum_{k=1}^{n}\left\|d_{k} \mid L_{2}\right\|^{2}=1$.

Let $l$ be such that $4^{l} \leq 16 n<4^{l+1}$ and $m:=4^{l}$. For $h=1,2 \ldots$ define

$$
\mathbb{F}_{h}:=\left\{k: \frac{1}{2^{h}}<\left\|d_{k} \mid L_{2}\right\| \leq \frac{2}{2^{h}}\right\} \quad \text { and } \quad \mathbb{F}:=\bigcup_{h=1}^{l} \mathbb{F}_{h} .
$$

First of all, we estimate the sum of all the differences with small norm:

$$
\begin{aligned}
\left\|\sum_{k \notin \mathbb{F}}\left[L_{2}, T\right] d_{k} \mid L_{2}\right\| & \leq \boldsymbol{\tau}\left(T \mid \mathcal{M}_{n}\right)\left(\sum_{k \notin \mathbb{F}}\left\|d_{k} \mid L_{2}\right\|^{2}\right)^{1 / 2} \\
& \leq \boldsymbol{\tau}\left(T \mid \mathcal{M}_{n}\right)\left(n 4^{-l}\right)^{1 / 2} \leq \frac{1}{2} \boldsymbol{\tau}\left(T \mid \mathcal{M}_{n}\right) .
\end{aligned}
$$

Here we used, that the sequence $\left(\boldsymbol{\tau}\left(T \mid \mathcal{M}_{n}\right)\right)$ is obviously non-decreasing and that $16 n<4^{l+1}$.

For the martingale difference sequence $\left(d_{k}\right)$ with $k \in \mathbb{F}$ we apply the glueing technique described above. Then it follows from (3.2) that for $k \in \mathbb{F}_{h}$

$$
\frac{1}{m 4^{h}}<\left\|\Phi_{j}^{m} d_{k}\left|L_{2}\left\|^{2}=\frac{1}{m}\right\| d_{k}\right| L_{2}\right\|^{2} \leq \frac{4}{m 4^{h}} .
$$

Therefore by disjointness, for any subset $\mathbb{L} \subseteq\{1, \ldots, m\}$ of cardinality $|\mathbb{L}|=$ $4^{h}$

$$
\frac{1}{m}<\left\|\sum_{i \in \mathbb{L}} \Phi_{i}^{m} d_{k} \mid L_{2}\right\|^{2} \leq \frac{4}{m} .
$$

Writing $\mathbb{L}_{j}^{(h)}:=\left\{4^{h}(j-1)+1, \ldots 4^{h} j\right\}$ and $\tilde{d}_{j}^{k}:=\sum_{i \in \mathbb{L}_{j}^{(h)}} \Phi_{i}^{m} d_{k}$ we obtain a martingale of length

$$
N:=\sum_{h=1}^{l} \sum_{k \in \mathbb{F}_{h}} 4^{l-h} \leq 4^{l} \sum_{h=1}^{l} \sum_{k \in \mathbb{F}_{h}}\left\|d_{k} \mid L_{2}\right\|^{2} \leq 4^{l} \leq 16 n .
$$

It follows from Lemma 3 that $\boldsymbol{\tau}^{\circ}\left(T \mid \mathcal{M}_{N}\right) \leq \boldsymbol{\tau}^{\circ}\left(T \mid \mathcal{M}_{16 n}\right)$ and therefore, we obtain from Lemma 2 that

$$
\begin{aligned}
\left\|\sum_{h=1}^{l} \sum_{k \in \mathbb{F}_{h}} \sum_{j=1}^{4^{l-h}}\left[L_{2}, T\right] \tilde{d}_{j}^{k} \mid L_{2}\right\| & \leq \boldsymbol{\tau}^{\circ}\left(T \mid \mathcal{M}_{N}\right) \sup \left\|\tilde{d}_{j}^{k} \mid L_{2}\right\| \sqrt{N} \\
& \leq \boldsymbol{\tau}^{\circ}\left(T \mid \mathcal{M}_{16 n}\right) \frac{2}{\sqrt{m}} 2^{l}=2 \boldsymbol{\tau}^{\circ}\left(T \mid \mathcal{M}_{16 n}\right) .
\end{aligned}
$$


This shows that

$$
\left\|\sum_{k \in \mathbb{F}}\left[L_{2}, T\right] d_{k} \mid L_{2}\right\| \leq 2 \boldsymbol{\tau}^{\circ}\left(T \mid \mathcal{M}_{16 n}\right) .
$$

Putting together (3.3) and (3.4) we obtain

$$
\boldsymbol{\tau}\left(T \mid \mathcal{M}_{n}\right) \leq \frac{1}{2} \boldsymbol{\tau}\left(T \mid \mathcal{M}_{n}\right)+2 \boldsymbol{\tau}^{\circ}\left(T \mid \mathcal{M}_{16 n}\right),
$$

which implies $\boldsymbol{\tau}\left(T \mid \mathcal{M}_{n}\right) \leq 4 \boldsymbol{\tau}^{\circ}\left(T \mid \mathcal{M}_{16 n}\right)$. Finally the assertion follows from the trivial fact that $\boldsymbol{\tau}^{\circ}\left(T \mid \mathcal{M}_{16 n}\right) \leq 4 \boldsymbol{\tau}^{\circ}\left(T \mid \mathcal{M}_{n}\right)$.

The summation operator $\Sigma: l_{1} \rightarrow l_{\infty}$ is defined by

$$
\Sigma\left(\xi_{k}\right):=\left(\sum_{h=1}^{k} \xi_{h}\right)
$$

while the finite summation operators $\Sigma_{n}: l_{1}^{n} \rightarrow l_{\infty}^{n}$ act between the finite dimensional spaces and are defined in the same way.

The significance of the ideal norms $\boldsymbol{\tau}^{\circ}\left(T \mid \mathcal{M}_{n}\right)$ is due to the following fact, which establishes the connection with the factorization of the finite summation operators.

Proposition 3. There exists a factorization $\Sigma_{n}=B_{n}\left[L_{2}, T\right] A_{n}$ of the finite summation operator $\Sigma_{n}$, such that $\left\|B_{n}\right\|\left\|A_{n}\right\| \leq 6 \sqrt{n} / \boldsymbol{\tau}^{\circ}\left(T \mid \mathcal{M}_{2 n}\right)$.

Proof. There is nothing to prove for $T=0$. For $T \neq 0$, by definition, for all $0<\delta<1$, there exists a sequence of martingale differences $d_{1}, \ldots, d_{2 n}$ such that $\left\|d_{k} \mid L_{2}\right\|=1$ and

$$
\left\|\sum_{k=1}^{2 n}\left[L_{2}, T\right] d_{k} \mid L_{2}\right\|>\delta \boldsymbol{\tau}^{\circ}\left(T \mid \mathcal{M}_{2 n}\right) \sqrt{2 n} .
$$

Choose $g^{\prime} \in\left[L_{2}, Y^{\prime}\right]$ such that $\left\|g^{\prime} \mid L_{2}\right\|=1$ and

$$
\left\langle\sum_{k=1}^{2 n}\left[L_{2}, T\right] d_{k}, g^{\prime}\right\rangle>\delta \boldsymbol{\tau}^{\circ}\left(T \mid \mathcal{M}_{2 n}\right) \sqrt{2 n} .
$$

Let

$$
\mathbb{F}:=\left\{k:\left\langle\left[L_{2}, T\right] d_{k}, g^{\prime}\right\rangle>\boldsymbol{\tau}^{\circ}\left(T \mid \mathcal{M}_{2 n}\right) \frac{\delta}{4 \sqrt{2 n}}\right\}
$$


It follows from Lemma 3 that

$$
\left\langle\sum_{k \in \mathbb{F}}\left[L_{2}, T\right] d_{k}, g^{\prime}\right\rangle \leq\left\|\sum_{k \in \mathbb{F}}\left[L_{2}, T\right] d_{k}\left|L_{2}\|\| g^{\prime}\right| L_{2}\right\| \leq \boldsymbol{\tau}^{\circ}\left(T \mid \mathcal{M}_{2 n}\right)|\mathbb{F}|^{1 / 2}
$$

and therefore

$$
\begin{aligned}
\delta \boldsymbol{\tau}^{\circ}\left(T \mid \mathcal{M}_{2 n}\right) \sqrt{2 n} & <\left\langle\sum_{k \in \mathbb{F}}\left[L_{2}, T\right] d_{k}, g^{\prime}\right\rangle+\sum_{k \notin \mathbb{F}}\left\langle\left[L_{2}, T\right] d_{k}, g^{\prime}\right\rangle \\
& \leq \boldsymbol{\tau}^{\circ}\left(T \mid \mathcal{M}_{2 n}\right)|\mathbb{F}|^{1 / 2}+2 n \boldsymbol{\tau}^{\circ}\left(T \mid \mathcal{M}_{2 n}\right) \frac{\delta}{4 \sqrt{2 n}} .
\end{aligned}
$$

This shows that $m:=|\mathbb{F}| \geq \delta^{2} 9 n / 8$. Choosing $\delta$ appropriately, we may arrange that $m \geq n$. In particular, we find elements $i_{1}<\cdots<i_{n}$ in $\mathbb{F}$.

We can now define $A_{n}: l_{1}^{n} \rightarrow\left[L_{2}, X\right]$ by

$$
A_{n} e_{k}:=\frac{d_{i_{k}}}{\left\langle\left[L_{2}, T\right] d_{i_{k}}, g^{\prime}\right\rangle}
$$

and $B_{n}:\left[L_{2}, Y\right] \rightarrow l_{\infty}^{n}$ by

$$
B_{n} f:=\left(\left\langle f, E_{i_{k}} g^{\prime}\right\rangle\right)_{k=1}^{n} .
$$

\section{Uniform convexity and smoothness and super weakly compact operators}

We now show, how the concepts above connect to the theory of weakly compact operators.

First of all, we repeat the classical definitions of uniform convexity and uniform smoothness of Banach spaces in the more general case of linear operators between Banach spaces. See e. g. Beauzamy [1, Def. 7, p. 121].

Definition. An operator $T: X \rightarrow Y$ is uniformly convex if for all $\varepsilon>0$ there exists $\delta>0$ such that

$$
\text { for }\left\|x_{ \pm}\right\|=1 \text { with } \frac{\left\|x_{+}+x_{-}\right\|}{2} \geq 1-\delta \text { it follows that } \frac{\left\|T x_{+}-T x_{-}\right\|}{2} \leq \varepsilon .
$$

The operator $T$ is uniformly smooth if for all $\varepsilon>0$ there exists $\delta>0$ such that

for $\|y\|=1$ and $\|x\| \leq \delta$ it follows that $\frac{\|y+T x\|+\|y-T x\|}{2} \leq 1+\varepsilon\|x\|$. 
Using an equivalent norm on the target space $Y$ does not spoil uniform convexity. This is, however, not the case for the source space $X$. Similarly, uniform smoothness depends on the special choice of the norm on the target space. We are therefore rather interested in uniformly convex renormable and uniformly smooth renormable operators.

Definition. An operator $T: X \rightarrow Y$ is uniformly convex renormable if there exists an equivalent norm \|\|$\cdot \|$ on $X$ such that $T:[X,\|\| \cdot\|\|] \rightarrow Y$ is uniformly convex.

An operator $T: X \rightarrow Y$ is uniformly smooth renormable if there exists an equivalent norm $\|\mid \cdot\|$ on $Y$ such that $T: X \rightarrow[Y,\||\cdot|\|]$ is uniformly smooth.

The above properties are equivalent to $T$ factoring through a uniformly convex or uniformly smooth Banach space, respectively; see [13, Prop. 7.10.11].

As in the case of Banach spaces, we can easily show that the concepts of uniform convexity and smoothness are dual to each other. See Lindenstrauss [11, Thm. 1] for a proof in the Banach space case, which can easily be carried over to linear operators.

Proposition 4. An operator $T: X \rightarrow Y$ is uniformly convex if and only if its dual $T^{\prime}: Y^{\prime} \rightarrow X^{\prime}$ is uniformly smooth.

The operator $T$ is uniformly smooth if and only if $T^{\prime}$ is uniformly convex.

Next we introduce super weakly compact operators, whose Banach space counterparts are the superreflexive Banach spaces, and show their connection with the factorization of the summation operators. Again, this basically follows from the, by now, classical proof that superreflexive Banach spaces do not factor the summation operators uniformly, cf. James [10], Beauzamy [2, Prop. 7, p. 236], or Heinrich [7, Thm. 5.1, p. 29].

Definition. An operator $T: X \rightarrow Y$ is weakly compact, if the image of the closed unit ball of $X$ under $T$ is relatively weakly compact in $Y$.

For the theory of ultraproducts of Banach spaces and linear operators, we refer to Heinrich's papers $[7,8]$. We mainly use the notation of Pietsch/ Wenzel [13].

Definition. An operator $T: X \rightarrow Y$ is super weakly compact, if all its ultrapowers $T^{\mathcal{U}}: X^{\mathcal{U}} \rightarrow Y^{\mathcal{U}}$ are weakly compact.

The summation operator is the typical non-weakly compact operator. Example 1. The summation operator $\Sigma$ is not weakly compact. 
Definition. An operator $T: X \rightarrow Y$ is said to factor the summation operator $\Sigma$, if there exist operators $A: l_{1} \rightarrow X$ and $B: Y \rightarrow l_{\infty}$ such that $\Sigma=B T A$.

The operator $T$ is said to factor the finite summation operators $\Sigma_{n}$ uniformly, if there exists a constant $c>0$, such that for all $n \in \mathbb{N}$ we can find factorizations $\Sigma_{n}=B_{n} T A_{n}$ such that $\left\|B_{n}\right\|\left\|A_{n}\right\| \leq c$.

The next proposition connects the above concepts.

Proposition 5. Let $T: X \rightarrow Y$, then the following properties are equivalent:

(i) $T$ is super weakly compact,

(ii) $T^{\mathcal{U}}$ does not factor the summation operator $\Sigma$ for any ultrafilter $\mathcal{U}$,

(iii) $T$ does not factor the finite summation operators $\Sigma_{n}$ uniformly.

Proof. The equivalence of (i) and (ii) is due to Lindenstrauss/Pełczyński $[12]$.

Assume that $T: X \rightarrow Y$ factors the finite summation operators uniformly and let $\mathcal{U}$ be any non-trivial ultrafilter on $\mathbb{N}$.

Let $J$ be the canonical map from $l_{1}$ into $l_{1}^{\mathcal{U}}$ induced by the map $x \mapsto$ $(x, x, \ldots)$. Note moreover, that the map $\left(x_{n}\right) \mapsto \mathrm{w}^{*}-\lim _{\mathcal{U}} x_{n}$ induces a well defined operator $Q$ from $l_{\infty}^{\mathcal{U}}$ onto $l_{\infty}$. The last fact is due to the weak-*compactness of the closed unit ball of $l_{\infty}$. Obviously $\|J\|=\|Q\|=1$.

By the assumption, there exists a constant $c$, such that we find for all $n \in \mathbb{N}$ operators $A_{n}: l_{1}^{n} \rightarrow X$ and $B_{n}: Y \rightarrow l_{\infty}^{n}$ satisfying $\left\|A_{n}\right\| \leq 1$, $\left\|B_{n}\right\| \leq c$, and $\Sigma_{n}=B_{n} T A_{n}$.

Denote furthermore by $J_{n}$ the canonical embedding of $l_{\infty}^{n}$ into $l_{\infty}$ and by $Q_{n}$ the projection from $l_{1}$ onto $l_{1}^{n}$.

We obtain a factorization of the summation operator $\Sigma$ via $T^{\mathcal{U}}$ as

$$
\Sigma=Q\left(J_{n} \Sigma_{n} Q_{n}\right)^{\mathcal{U}} J=Q\left(J_{n} B_{n}\right)^{\mathcal{U}} T^{\mathcal{U}}\left(A_{n} Q_{n}\right)^{\mathcal{U}} J
$$

This proves that (ii) $\Rightarrow$ (iii).

If on the other hand (ii) does not hold, then $T^{\mathcal{U}}$ factors $\Sigma$ for some ultrafilter $\mathcal{U}$. In the sense of Heinrich [7, Def. 1.1, p. 7], $T^{\mathcal{U}}$ is finitely representable in $T$, i. e. for all $\varepsilon>0$ and each finite dimensional subspace $\widehat{X}_{0} \subseteq X^{\mathcal{U}}$ and finite codimensional subspace $\widehat{Y}_{0} \subseteq Y^{\mathcal{U}}$ there are a finite dimensional subspace $X_{0} \subseteq X$ and a finite codimensional subspace $Y_{0} \subseteq Y$ and maps $R: \widehat{X}_{0} \rightarrow X_{0}$ and $S: Y / Y_{0} \rightarrow Y^{\mathcal{U}} / \widehat{Y}_{0}$ such that

$$
\left\|\widehat{Q} T^{\mathcal{U}} \widehat{J}-S Q T J R\right\| \leq \varepsilon
$$


where $J$ and $\widehat{J}$ are the canonical embedding maps of $X_{0}$ and $\widehat{X}_{0}$ and $Q$ and $\widehat{Q}$ are the canonical quotient maps of $Y_{0}$ and $\widehat{Y}_{0}$.

It follows, that for all $n \in \mathbb{N}$ there is a subspace $X_{0} \subseteq X$ and a subspace $Y_{0} \subseteq Y$ and operators $R: l_{1}^{n} \rightarrow X_{0}$ and $S: Y / Y_{0} \rightarrow l_{\infty}^{n}$, such that

$$
\left\|\Sigma_{n}-S Q T J R\right\| \leq \varepsilon .
$$

Moreover, since $\Sigma_{n}$ is injective, by the remark following Definition 1.1 in [7, p. 8], we can even arrange that $\varepsilon=0$. This implies that $T$ factors $\Sigma_{n}$ uniformly.

It was shown in Beauzamy [1, Thm. I.1, p. 111] that uniformly convex renormability and super weak compactness are in fact equivalent properties. See also Heinrich's paper [7, Thm. 5,p. 29] and the detailed presentation in Pietsch/Wenzel [13, Sect. 7.6].

One obtains the following equivalences.

Proposition 6. Let $T: X \rightarrow Y$, then the following properties are equivalent:

(i) $T$ is super weakly compact,

(ii) $T$ is uniformly convex renormable,

(iii) $T$ does not factor the finite summation operators $\Sigma_{n}$ uniformly.

\section{Main theorem}

Before we can formulate and prove the main theorem some more preparations are required.

The following classical result provides the missing link between the operators $T$ and $\left[L_{2}, T\right]$. It was first proved by Day [5, Thm. 2, p. 504] for Banach spaces, but Day's proof can straightforwardly be extended to the operator case.

Proposition 7. The operator $T$ is uniformly convex if and only if $\left[L_{2}, T\right]$ is uniformly convex.

Finally, we will need the Haar cotype ideal norms of the finite summation operators.

Example 2. $\quad \frac{1}{2}(n+1)^{1 / 2} \leq \boldsymbol{\gamma}_{n}\left(\Sigma_{2^{n}} \mid \mathcal{H}\left(\mathbb{D}_{0}^{n}\right)\right) \leq(n+1)^{1 / 2}$. 
Proof. Obviously, for any operator $T: X \rightarrow Y$

$$
\begin{aligned}
\left\|\sum_{\mathbb{D}_{0}^{n}} T x_{k}^{(j)} \chi_{k}^{(j)} \mid L_{2}\right\| & \leq(n+1)^{1 / 2}\left(\sum_{k=0}^{n}\left\|\sum_{j=1}^{2^{k-1}} T x_{k}^{(j)} \chi_{k}^{(j)} \mid L_{2}\right\|^{2}\right)^{1 / 2} \\
& =(n+1)^{1 / 2}\left(\sum_{k=0}^{n} \sum_{j=1}^{2^{k-1}}\left\|T x_{k}^{(j)}\right\|^{2}\left\|\chi_{k}^{(j)} \mid L_{2}\right\|^{2}\right)^{1 / 2} \\
& \leq(n+1)^{1 / 2}\|T\|\left(\sum_{\mathbb{D}_{0}^{n}}\left\|x_{k}^{(j)}\right\|^{2}\right)^{1 / 2}
\end{aligned}
$$

where we have used that for fixed $k$ the Haar functions $\chi_{k}^{(1)}, \ldots, \chi_{k}^{\left(2^{k-1}\right)}$ have disjoint support. This proves the upper estimate by virtue of Proposition 2.

To see the lower estimate, let $f:[0,1) \rightarrow l_{1}^{2^{n}}$ be defined by

$$
f(t):=e_{i} \quad \text { if } t \in \Delta_{n}^{(i)},
$$

where $e_{i}$ is the $i$-th unit vector in $l_{1}^{2^{n}}$. Write $f$ as a Haar polynomial

$$
f=\sum_{\mathbb{D}_{0}^{n}} x_{k}^{(j)} \chi_{k}^{(j)}, \quad \text { where } \quad x_{k}^{(j)}=\int_{0}^{1} f(t) \chi_{k}^{(j)}(t) d t .
$$

Obviously

$$
\left\|f\left|L_{2}\|=\| \sum_{\mathbb{D}_{0}^{n}} x_{k}^{(j)} \chi_{k}^{(j)}\right| L_{2}\right\|=\left(\frac{1}{2^{n}} \sum_{i=1}^{2^{n}}\left\|e_{i}\right\|^{2}\right)^{1 / 2}=1 .
$$

Writing $\mathbb{N}_{k}^{(j)}:=\left\{i: \Delta_{n}^{(i)} \subseteq \Delta_{k}^{(j)}\right\}$, it follows that

$$
x_{k}^{(j)}=\frac{1}{2^{n}} 2^{(k-1) / 2}(0, \ldots, 0 \underbrace{+1, \ldots,+1}_{\mathbb{N}_{k}^{(2 j-1)}}, \underbrace{-1, \ldots,-1}_{\mathbb{N}_{k}^{(2 j)}}, 0, \ldots, 0)
$$

and

$$
\Sigma_{2^{n}} x_{k}^{(j)}=\frac{1}{2^{n}} 2^{(k-1) / 2}(0, \ldots, 0 \underbrace{1,2, \ldots, 2^{n-k}}_{\mathbb{N}_{k}^{(2 j-1)}}, \underbrace{2^{n-k}-1, \ldots, 1,0}_{\mathbb{N}_{k}^{(2 j)}}, 0, \ldots, 0),
$$

which in turn yields $\left\|\Sigma_{2^{n}} x_{k}^{(j)}\right\|=2^{-(k+1) / 2}$. Hence

$$
\left(\sum_{\mathbb{D}_{0}^{n}}\left\|\Sigma_{2^{n}} x_{k}^{(j)}\right\|^{2}\right)^{1 / 2}=\frac{1}{2}(n+1)^{1 / 2},
$$

which proves the assertion. 
We are now ready to state our most important result.

Theorem 2. For an operator $T: X \rightarrow Y$ the following properties are equivalent:

(i) T has martingale subtype,

(ii) T has martingale subcotype,

(iii) Thas Haar subtype,

(iv) $T$ has Haar subcotype,

(v) $T$ is super weakly compact,

(vi) $T$ does not factor the finite summation operators $\Sigma_{n}$ uniformly,

(vii) $T$ is uniformly convex renormable,

(viii) $\left[L_{2}, T\right]$ is uniformly convex renormable,

(ix) $T$ is uniformly smooth renormable.

Proof The equivalence of (i)-(iv) was shown in Proposition 1. The equivalence of (v)-(vii) was shown in Proposition 6. Proposition 7 shows that (vii) and (viii) are equivalent, hence all the properties (v)-(vii) for $T$ and (v)-(vii) for $\left[L_{2}, T\right]$ are equivalent.

Assume that (i) does not hold. Then for some $c>0$ there are infinitely many numbers $n$, such that

$$
\frac{\boldsymbol{\tau}\left(T \mid \mathcal{M}_{n}\right)}{\sqrt{n}} \geq c
$$

It follows from Lemma 4 that for these numbers

$$
\frac{6 \sqrt{n}}{\boldsymbol{\tau}^{\circ}\left(T \mid \mathcal{M}_{2 n}\right)} \leq \frac{6 \sqrt{n}}{\boldsymbol{\tau}\left(T \mid \mathcal{M}_{2 n}\right)} \leq \frac{6}{c}
$$

and therefore, by Proposition 3 there exist factorizations $\Sigma_{n}=B_{n}\left[L_{2}, T\right] A_{n}$ such that $\left\|B_{n}\right\|\left\|A_{n}\right\| \leq 6 / c$. Of course, this yields such factorizations for all $n \in \mathbb{N}$ and therefore (vi) cannot hold for $\left[L_{2}, T\right]$.

Hence we have shown that (vi) implies (i). On the other hand Example 2 shows that (iv) implies (vi).

So far, we have shown that (i)-(viii) are equivalent. Finally, since by Proposition 2 properties (i)-(iv) for $T$ are equivalent to the same properties for $T^{\prime}$, it follows from Proposition 4 that also (ix) is equivalent to (vii) and hence to all other properties.

Remark. Note that Pisier's Theorem 1 can easily be obtained from our Theorem 2 using the submultiplicativity of the ideal norms $\boldsymbol{\tau}\left(I_{X} \mid \mathcal{M}_{n}\right)$. Namely, 
it follows from $\boldsymbol{\tau}\left(I_{X} \mid \mathcal{M}_{n}\right)=o\left(n^{1 / 2}\right)$ and the submultiplicativity, that there is a constant $c$ and a number $p_{0}>1$ such that

$$
\boldsymbol{\tau}\left(I_{X} \mid \mathcal{M}_{n}\right) \leq c n^{1 / p_{0}-1 / 2} \quad \text { for all } n \in \mathbb{N} .
$$

By Pisier [14], this implies that $X$ has martingale type $p$ for all $p<p_{0}$.

To formulate the last example, we will need one more definition.

Definition. For $T: X \rightarrow Y$, the Haar type $p$ ideal norm $\boldsymbol{\tau}_{p}\left(T \mid \mathcal{H}\left(\mathbb{D}_{1}^{n}\right)\right)$, is the smallest constant $c \geq 0$ such that

$$
\left\|\sum_{\mathbb{D}_{1}^{n}}\left[L_{2}, T\right] x_{k}^{(j)} \chi_{k}^{(j)} \mid L_{p}\right\| \leq c\left(\sum_{k=1}^{n}\left\|\sum_{j=1}^{2^{k-1}} x_{k}^{(j)} \chi_{k}^{(j)} \mid L_{p}\right\|^{p}\right)^{1 / p}
$$

for all $\left(x_{k}^{(j)}\right) \subseteq X$

In particular, $\boldsymbol{\tau}_{2}\left(T \mid \mathcal{H}\left(\mathbb{D}_{1}^{n}\right)\right)=\boldsymbol{\tau}\left(T \mid \mathcal{H}\left(\mathbb{D}_{1}^{n}\right)\right)$. An operator $T$ is said to have martingale type $p$ if the sequence $\left(\boldsymbol{\tau}_{p}\left(T \mid \mathcal{H}\left(\mathbb{D}_{1}^{n}\right)\right)\right)$ is bounded.

The following example shows that an analogue of the Davis-FigielJohnson-Pełczyński Theorem on weakly compact operators (they factor through a reflexive Banach space; see [4, Cor. 1, p. 314]) cannot hold for super weakly compact operators, since if $T$ factors through a superreflexive Banach space then it follows already that it has martingale type $p$ for some $p>1$; see [14, Thm. 3.2, p. 340].

Let $t=\left(\tau_{n}\right)$ be a non-increasing sequence of positive numbers. We consider the diagonal operator $D_{t}: l_{1} \rightarrow l_{1}$ defined by $D_{t}\left(\xi_{k}\right):=\left(\tau_{k} \xi_{k}\right)$.

The following fact is proved in [15].

Example 3. $\quad \boldsymbol{\tau}_{p}\left(D_{t} \mid \mathcal{H}\left(\mathbb{D}_{1}^{n}\right)\right)=\left(\sum_{k=1}^{n}\left|\tau_{k}\right|^{p^{\prime}}\right)^{1 / p^{\prime}}$.

Corollary. If $\tau_{k}=1 /(1+\log k)$ then the operator $D_{t}$ is super weakly compact but does not have martingale type $p$ for any $p>1$ and hence does not factor through a superreflexive Banach space.

\section{References}

[1] Beauzamy, B., Opérateurs uniformément convexifiants. Studia Math. 57 (1976), 103-139.

[2] Beauzamy, B., Introduction to Banach spaces and their geometry. NorthHolland Mathematics Studies 68. North-Holland, second edition, 1985. 
[3] Bourgain, J., Kalton, N., and Tzafriri, L., Geometry of finite dimensional subspaces and quotients of $L_{p}$. In Geometric aspects of functional analysis, Israel 1987-88. Lect. Notes in Math. 1376, 138-175, 1989.

[4] Davis, W., Figiel, T., Johnson, W. B., and Peeczyński, A., Factoring weakly compact operators. J. Func. Anal. 17 (1974), 311-327.

[5] Day, M. M., Some more uniformly convex spaces. Bull. Amer. Math. Soc. 47 (1941), 504-507.

[6] Enflo, P., Banach spaces which can be given an equivalent uniformly convex norm. Israel. J. Math. 13 (1972), no. 3-4, 281-288.

[7] Heinrich, S., Finite representability and super-ideals of operators. Dissertationes Math. (Rozprawy Mat.) 172 (1980), 37 p.

[8] Heinrich, S., Ultraproducts in Banach space theory. J. Reine Angew. Math. 313 (1980), 72-104.

[9] Hinrichs, A., Operators of Rademacher and Gaussian subcotype. Forschungsergebnisse der FSU Jena.

[10] James, R. C., Super-reflexive Banach spaces. Canad. J. Math. 24 (1972), no. $5,896-904$.

[11] Lindenstrauss, J., On the modulus of smoothness and divergent series in Banach spaces. Michigan. Math. J. 10 (1963), 241-252.

[12] Lindenstrauss, J. And Pęczyński, A., Absolutely summing operators in $\mathcal{L}_{p}$-spaces and applications. Studia Math. 29 (1968), 275-326.

[13] Pietsch, A. And Wenzel, J., Orthonormal systems and Banach space geometry. Cambridge University Press, 1998.

[14] Pisier, G., Martingales with values in uniformly convex spaces. Israel $\mathrm{J}$. Math. 20 (1975), no. 3-4, 326-350.

[15] Wenzel, J., Haar type ideal norms of diagonal operators. Preprint. http:

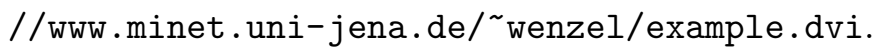

Recibido: 3 de julio de 2000

Jörg Wenzel

Mathematical Institute Friedrich-Schiller-University Jena

07740 Jena

Germany

wenzel@minet.uni-jena.de 